\title{
Particle-accelerator constraints on isotropic modifications of the speed of light
}

\author{
Michael A. Hohensee, ${ }^{1}$ Ralf Lehnert, ${ }^{2,3}$ David F. Phillips, ${ }^{4}$ and Ronald L. Walsworth ${ }^{1,4}$ \\ ${ }^{1}$ Department of Physics, Harvard University, Cambridge, Massachusetts, 02138, USA \\ ${ }^{2}$ Max-Planck-Institut für Physik, Föhringer Ring 6, 80805 München, Germany \\ ${ }^{3}$ Instituto de Ciencias Nucleares, Universidad Nacional Autónoma de México, A. Postal 70-543, 04510 México D.F., Mexico \\ ${ }^{4}$ Harvard-Smithsonian Center for Astrophysics, Cambridge, Massachusetts 02138, USA
}

(Dated: October 30, 2018)

\begin{abstract}
The absence of vacuum Cherenkov radiation for $104.5 \mathrm{GeV}$ electrons and positrons at LEP combined with the observed stability of $300 \mathrm{GeV}$ photons at the Tevatron constrains deviations of the speed of light relative to the maximal attainable speed of electrons. Within the Standard-Model Extension (SME), the limit $-5.8 \times 10^{-12} \leq \tilde{\kappa}_{\mathrm{tr}}-\frac{4}{3} c_{e}^{00} \leq 1.2 \times 10^{-11}$ is extracted, which sharpens previous bounds by more than 3 orders of magnitude. The potential for further refinements of this limit with terrestrial experiments and astrophysical observations is discussed.
\end{abstract}

PACS numbers: 11.30.Cp, 12.20.-m, 41.60.Bq, 29.20.-c

The speed of light, $c$, has played a crucial role in both the conception of Special and General Relativity and its experimental tests. The confidence we place in Relativity theory is embodied in the fact that $c \equiv 299792458 \mathrm{~m} \cdot \mathrm{s}^{-1}$ is set to be a constant and provides the basis for the definition of length in the International System of Units.

Currently, Relativity tests, including precise searches for modifications of the speed of light, are experiencing a revival of interest, motivated by theoretical studies that identify minute violations of Lorentz symmetry as a promising imprint of Planck-scale physics [1]. A general theoretical description of weak Lorentz symmetry breaking at low energies is provided by the Standard-Model Extension (SME), which contains the usual Standard Model and General Relativity as limiting cases [2, 3]. To date, the SME has served as the basis for numerous Relativity tests in many physical systems [4, 5, 6].

The majority of potential Relativity violations in electrodynamics is governed by the dimensionless $\left(k_{F}\right)^{\alpha \beta \gamma \delta}$ coefficient in the SME, which causes the speed of light to be direction- and polarization-dependent. The birefringent components of $k_{F}$ are bounded down to $10^{-37}$ with spectropolarimetric studies of astrophysical sources [7]. The remaining components $\tilde{\kappa}_{e-}, \tilde{\kappa}_{o+}$, and $\tilde{\kappa}_{\text {tr }}$ are respectively the symmetric, antisymmetric, and trace pieces of a $3 \times 3$ matrix; they lead to polarization-independent shifts of the speed of light. These parameters can be bounded with Michelson-Morley experiments [8], for which effects of $\tilde{\kappa}_{e-}$ are unsuppressed, $\tilde{\kappa}_{o+}$ effects are suppressed by $\beta$, and $\tilde{\kappa}_{\mathrm{tr}}$ effects are suppressed by $\beta^{2}[9]$, where $\beta \simeq 10^{-4}$ is the Earth's orbital speed. The corresponding limits are $10^{-17}, 10^{-13}$, and $10^{-8}$, respectively.

These results indicate that improvements of limits on $\tilde{\kappa}_{\text {tr }}$ assume particular urgency. Here, we use the analogy

$$
n=1+\tilde{\kappa}_{\mathrm{tr}}+\mathcal{O}\left(\tilde{\kappa}_{\mathrm{tr}}^{2}\right)
$$

between $\tilde{\kappa}_{\text {tr }}$ and a conventional frequency-independent refractive index $n$ to reduce the sensitivity gap between $\tilde{\kappa}_{\text {tr }}$ and $\tilde{\kappa}_{o+}$. A positive $\tilde{\kappa}_{\text {tr }}$ would imply $n>1$, so that the maximal attainable speed (MAS) of other particles can exceed the speed of light. This allows charges in a Lorentz-violating $\left(\tilde{\kappa}_{\text {tr }}>0\right)$ vacuum to move faster than the modified speed of light $c / n$ and to become unstable against the emission of Cherenkov photons. A negative $\tilde{\kappa}_{\text {tr }}$ would imply that the MAS of charges is now less than the speed of light [10]. In this respect, the roles of the photon and the charge are reversed relative to the $\tilde{\kappa}_{\text {tr }}>0$ case suggesting that then the photon is unstable. Indeed, a simple argument shows that above some energy threshold photon decay into a charge-anticharge pair is now kinematically allowed. We employ the absence of these two effects for electrons at LEP and photons at the Tevatron to derive improved limits at the $10^{-11}$ level on $\tilde{\kappa}_{\text {tr }}-\frac{4}{3} c_{e}^{00}$, a quantity that describes differences between the speed of light and the electron's MAS.

The quality of such an analysis rests on various requirements. First, the nature of the charge must be known: its MAS serves as the reference relative to which the speed of light is constrained [12]. Second, the total rates for vacuum Cherenkov radiation and photon decay must be known. Purely kinematical analyses of energymomentum conservation in these processes are not themselves sufficient if we are to draw conclusions based on their absence [13, 14]. Third, the effects of other types of Lorentz violation must be considered: vacuum Cherenkov radiation and photon decay could also be generated by, e.g., the electron's SME coefficients. Note that SME coefficients can typically not be set to zero because they may be generated by quantum effects. A fourth important factor is a minimal amount of modeling required to extract the bound. For example, cosmic-ray analyses of Lorentz violation necessitate varying degrees of astrophysical and shower-development modeling.

The analysis reported here incorporates all four of the above requirements, providing a clean and conservative bound on $\tilde{\kappa}_{\mathrm{tr}}-\frac{4}{3} c_{e}^{00}$. Our study simultaneously takes advantage of the high-quality data collected at the world's highest-energy accelerators, as well as their superbly con- 
trolled laboratory environments. In so doing, we both improve upon previous constraints, and highlight new avenues for exploring SME physics at existing and future colliders.

Although related tests using observations of ultrahighenergy cosmic-rays (UHECR) have also sought to constrain Lorentz violating modifications of the fermionphoton vertex 15, 16, 17, 18, 19, 20, 21, 22], their conclusions are not directly comparable to our result. Many UHECR studies do not estimate the rate of vacuum Cherenkov radiation or photon decay, an issue that is nontrivial even for propagation over cosmological distances [14]; or they focus on dispersion-relation parameters whose relation to $\tilde{\kappa}_{\text {tr }}$ is unknown or unclear [15, 16, 17, 18]. More recent studies 21, 22] involve atomic nuclei as well as electrons, and thus constrain $\tilde{\kappa}_{\mathrm{tr}}-\frac{4}{3} c_{X}^{00}$ for the generally undetermined nucleus $X$ that serves as the UHECR primary scatterer. Quantitative interpretation of such studies in the broader context of the SME is complicated by both the composite nature of the nuclei as well as the contribution of numerous other SME coefficients that cannot be ignored at UHECR energy scales. Finally, some UHECR investigations of Lorentz-symmetry violation require various mild assumptions regarding astrophysical processes [19, 20].

The physical system we will consider consists of photons and electrons, so we begin by recalling the singleflavor QED limit of the flat-spacetime SME [2]:

$$
\begin{aligned}
\mathcal{L}= & -\frac{1}{4} F^{2}-\frac{1}{4}\left(k_{F}\right)^{\kappa \lambda \mu \nu} F_{\kappa \lambda} F_{\mu \nu}+\left(k_{A F}\right)^{\mu} A^{\nu} \tilde{F}_{\mu \nu} \\
& +\frac{1}{2} i \bar{\psi} \Gamma^{\nu} \stackrel{\leftrightarrow}{D}_{\nu} \psi-\bar{\psi} M \psi
\end{aligned}
$$

where

$$
\begin{aligned}
\Gamma^{\nu} & \equiv \gamma^{\nu}+c_{e}^{\mu \nu} \gamma_{\mu}+d_{e}^{\mu \nu} \gamma_{5} \gamma_{\mu}, \\
M & \equiv m_{e}+b_{e}^{\mu} \gamma_{5} \gamma_{\mu}+\frac{1}{2} H_{e}^{\mu \nu} \sigma_{\mu \nu} .
\end{aligned}
$$

Here, $F^{\mu \nu}=\partial_{\mu} A_{\nu}-\partial_{\nu} A_{\mu}$ is the electromagnetic fieldstrength tensor and $\tilde{F}^{\mu \nu}=\frac{1}{2} \epsilon^{\mu \nu \rho \sigma} F_{\rho \sigma}$ denotes its dual. The spinor $\psi$ describes electrons of mass $m_{e}$, and the usual $\mathrm{U}(1)$-covariant derivative is denoted by $D^{\mu}=$ $\partial^{\mu}+i e A^{\mu}$. The spacetime-independent SME coefficients $\left(k_{F}\right)^{\mu \nu \rho \lambda},\left(k_{A F}\right)^{\mu}, b^{\mu}, c^{\mu \nu}, d^{\mu \nu}$, and $H^{\mu \nu}$ control the extent of Lorentz and CPT violation.

In what follows, we are primarily interested in the $\tilde{\kappa}_{\text {tr }}$ component of the CPT-even $\left(k_{F}\right)^{\mu \nu \rho \lambda}$. The $k_{F}$ coefficient exhibits the symmetries of the Riemann tensor, and its double trace $\left(k_{F}\right)^{\mu \nu}{ }_{\mu \nu}$ vanishes. This leaves 19 independent components. In a given coordinate system, which is conventionally chosen to be the Sun-centered celestial equatorial frame, $k_{F}$ can be decomposed as follows [23]. Ten components are associated with birefringence and can be grouped into the two dimensionless $3 \times 3$ matrices $\tilde{\kappa}_{o-}$ and $\tilde{\kappa}_{e+}$. The remaining nine components

$$
\tilde{k}^{\mu \nu} \equiv\left(k_{F}\right)_{\alpha}^{\mu \alpha \nu}
$$

give rise to $\tilde{\kappa}_{e-}, \tilde{\kappa}_{o+}$, and $\tilde{\kappa}_{\text {tr }}$, as explained earlier. In particular, $\tilde{\kappa}_{\mathrm{tr}}=-\frac{2}{3}\left(k_{F}\right)^{0 j 0 j}$, where the index $j$ runs from 1 to 3 and is summed over in this expression.

All of the SME coefficients in Lagragian (2) modify either the photon's or the electron's dispersion relation and therefore also the kinematics of the electron-photon vertex. It follows that $\tilde{\kappa}_{\text {tr }}$ cannot be singled out in studies of vacuum Cherenkov radiation and photon decay; the other SME coefficients for the photon and the electron must in general also be taken into account. However, a careful analysis of previous experiments reveals existing stringent limits on these additional SME parameters [12]. The scale of these limits is governed by

$$
\mathcal{S} \equiv \max \left(\tilde{\kappa}_{e-}, \tilde{\kappa}_{e+}, \tilde{\kappa}_{o+}, \tilde{\kappa}_{o-}, \frac{k_{A F}}{m_{e}}, \frac{b}{m_{e}}, c, d, \frac{H}{m_{e}}\right),
$$

where the absolute values of the individual components listed here are implied. At present, $\mathcal{S} \sim 10^{-13}$ is dominated by $\tilde{\kappa}_{o+}$ [5]. This value is more than five orders of magnitude smaller than present limits on $\tilde{\kappa}_{\text {tr }}$, and about a factor of $10^{2}$ smaller than the bound on $\tilde{\kappa}_{\text {tr }}$ we derive here. We therefore can safely ignore other SME coefficients in our analysis and retain $\tilde{\kappa}_{\text {tr }}$ only.

A related issue concerns the physical equivalence of the photon's $\tilde{k}^{\mu \nu}$ and electron's $c_{e}^{\mu \nu}$. These two coefficients are associated with the same phenomenology, and they can therefore not be distinguished within the framework of Lagragian (2). In the present context, our $\tilde{\kappa}_{\text {tr }}$ model is physically equivalent to a model with

$$
c_{e}^{00}=-\frac{3}{4} \tilde{\kappa}_{\mathrm{tr}} \quad \text { and } \quad c_{e}^{j j}=-\frac{1}{4} \tilde{\kappa}_{\mathrm{tr}} .
$$

In this expression, there is no sum over $j=1,2,3$. This equivalence can be established rigorously with a coordinate rescaling [23, 24], which implies that only $\tilde{\kappa}_{\mathrm{tr}}-\frac{4}{3} c_{e}^{00}$ is observable in the context of Lagrangian (2). This rescaling can be used to remove either $\tilde{\kappa}_{\text {tr }}$ or $c_{e}^{00}$ from the model. We often select coordinates such that $c_{e}^{00}=0$, but undo this special rescaling and reinstate $c_{e}^{00}$ when quoting results.

At leading order, the photon dispersion relation in the presence of $\tilde{\kappa}_{\text {tr }}$ is given by [23]

$$
E_{\gamma}^{2}-\left(1-\tilde{\kappa}_{\mathrm{tr}}\right) \vec{p}^{2}=0,
$$

where $p^{\mu} \equiv\left(E_{\gamma}, \vec{p}\right)$ is the photon's 4-momentum. Thus, the speed of light is $\left(1-\tilde{\kappa}_{\mathrm{tr}}\right)$, and in the present context, in which we treat the fermion dispersion relation as being unaffected by Lorentz violation, vacuum Cherenkov radiation can only occur for positive $\tilde{\kappa}_{\text {tr }}$. Using Eq. (7) and energy-momentum conservation for the emission of a Cherenkov photon yields the energy threshold [19]

$$
E_{\mathrm{VCR}}=\frac{1-\tilde{\kappa}_{\mathrm{tr}}}{\sqrt{\left(2-\tilde{\kappa}_{\mathrm{tr}}\right) \tilde{\kappa}_{\mathrm{tr}}}} m_{e}=\frac{m_{e}}{\sqrt{2 \tilde{\kappa}_{\mathrm{tr}}}}+\mathcal{O}\left(\sqrt{\tilde{\kappa}_{\mathrm{tr}}}\right) \text {. }
$$

For electrons with energies above $E_{\mathrm{VCR}}$, vacuum Cherenkov radiation is kinematically allowed. Equation (8) can alternatively be derived from the usual 
Cherenkov condition that the electron must be faster than the speed of light $\left(1-\tilde{\kappa}_{\text {tr }}\right)$.

We extract a limit on $\tilde{\kappa}_{\text {tr }}$ through the absence of observed vacuum Cherenkov radiation. This requires the emission to be efficient enough that charges with energies above $E_{\mathrm{VCR}}$ are rapidly decelerated below threshold. Near $E_{\mathrm{VCR}}$, the dominant deceleration process is single-photon emission with the estimated rate [19]

$$
\Gamma_{\mathrm{VCR}}=\alpha m_{e}^{2} \frac{\left(E_{e}-E_{\mathrm{VCR}}\right)^{2}}{2 E_{e}^{3}},
$$

where $\alpha$ is the fine-structure constant, and $E_{e}$ denotes the electron energy. This expression shows that the emission process is quite efficient, and we now use it to derive limits on $\tilde{\kappa}_{\text {tr }}$ from the energies attained by primary electrons at the LEP collider. The highest laboratory-frame particle energy reached at LEP was $E_{\mathrm{LEP}}=104.5 \mathrm{GeV}$ with a relative uncertainty in the center-of-mass energy $\Delta E_{\mathrm{CM}} / E_{\mathrm{CM}}$ below $2.0 \times 10^{-4}$ [25]. Using Eq. (9), we find that if $E_{\mathrm{VCR}}=104 \mathrm{GeV}$, electrons initially accelerated to $104.5 \mathrm{GeV}$ would be rapidly slowed by emission of Cherenkov photons to an energy below $E_{\mathrm{VCR}}$ over a $1 / e$ length of about $95 \mathrm{~cm}$. The total energy lost to the Cherenkov effect in such a scenario would far exceed the value allowed by measurements [12]. With Eq. (8), the requirement that $E_{\mathrm{VCR}}$ be greater than $104 \mathrm{GeV}$ becomes

$$
\tilde{\kappa}_{\mathrm{tr}}-\frac{4}{3} c_{e}^{00} \leq 1.2 \times 10^{-11},
$$

where we include the dependence on $c_{e}^{00}$. This bound is significantly smaller than previous laboratory limits on $\tilde{\kappa}_{\text {tr }}$. Note also that the scale $\mathcal{S}$ defined in Eq. (15) is not yet reached, which justifies the exclusion of other photon or electron SME coefficients in our analysis.

For negative $\tilde{\kappa}_{\mathrm{tr}}<0$, the dispersion relation (7) remains valid, and photons may travel faster than the MAS of electrons [10]. This precludes vacuum Cherenkov radiation at the cost of eliminating photon stability: for $E_{\gamma}$ above the threshold

$$
E_{\mathrm{pair}}=\frac{2 m_{e}}{\sqrt{\tilde{\kappa}_{\mathrm{tr}}\left(\tilde{\kappa}_{\mathrm{tr}}-2\right)}}=\sqrt{\frac{2}{-\tilde{\kappa}_{\mathrm{tr}}}} m_{e}+\mathcal{O}\left(\sqrt{\tilde{\kappa}_{\mathrm{tr}}}\right),
$$

photon decay into an electron-positron pair is kinematically allowed [12, 16]. The corresponding leading-order decay rate is given by [12, 22]

$$
\Gamma_{\text {pair }}=\frac{2}{3} \alpha E_{\gamma} \frac{m_{e}^{2}}{E_{\text {pair }}^{2}} \sqrt{1-\frac{E_{\text {pair }}^{2}}{E_{\gamma}^{2}}}\left(2+\frac{E_{\text {pair }}^{2}}{E_{\gamma}^{2}}\right) .
$$

Paralleling the Cherenkov case, this process is also highly efficient. For example, a $40 \mathrm{GeV}$ photon with energy $1 \%$ above threshold would decay after traveling about $30 \mu \mathrm{m}$.

The above reasoning establishes that the existence of long-lived photons with high energies constrains negative values of $\tilde{\kappa}_{\text {tr }}$. Photons generated in terrestrial laboratories provide a clean, well-characterized source for bounding $\tilde{\kappa}_{\text {tr }}$. Although the accessible energies are lower than those in cosmic rays, terrestrial tests offer larger data samples and a better control of the experimental conditions. Hadron colliders produce the highest-energy photons and therefore yield tight Earth-based experimental limits on $\tilde{\kappa}_{\text {tr }}$. Thus, we consider Fermilab's Tevatron $p \bar{p}$ collider with center-of-mass energies up to $1.96 \mathrm{TeV}$. Isolated-photon production with an associated jet is important to QCD studies and has been investigated with the D0 detector. The recorded photon spectrum extended up to a single event at $442 \mathrm{GeV}$ [26], which would imply $\tilde{\kappa}_{\text {tr }} \gtrsim-3 \times 10^{-13}$. While such a single event is insufficient to draw conservative conclusions regarding Lorentz symmetry, it is indicative of the sensitivity of this method.

We restrict our analysis to lower-energy D0 photon data with good statistics, where photons with energies up to $340.5 \mathrm{GeV}$ were observed [27], and comparisons to QCD theory were made. The $340.5 \mathrm{GeV}$ bin extended from $300 \mathrm{GeV}$ to $400 \mathrm{GeV}$; the measured flux deviated by a factor of $0.52 \pm 0.26$ from QCD predictions [27], so that at most $74 \%$ of the produced photon flux can be lost due to hypothetical photon decay. We continue by conservatively assuming that all events in this bin were $300 \mathrm{GeV}$ photons, and we take $E_{\text {pair }}=300 \mathrm{GeV}$. This is again justified by the rapid photon-decay rate (12): if $E_{\text {pair }}$ were just $0.1 \mathrm{keV}$ below the lowest observed $300 \mathrm{GeV}$ photon energy, the photon deficit would be larger than the allowed $74 \%$ [12]. In other words, the uncertainty in $E_{\text {pair }}$ is essentially determined by the resolution of the photonenergy measurement. This reasoning gives the limit

$$
-5.8 \times 10^{-12} \leq \tilde{\kappa}_{\mathrm{tr}}-\frac{4}{3} c_{e}^{00},
$$

where we again include the contribution of $c_{e}^{00}$. Like the Cherenkov constraint, the photon-stability limit (13) is larger than the scale $\mathcal{S}$, so other photon- or electronsector coefficients are not further constrained by this argument. At the same time, this justifies the exclusion of these additional coefficients from our study.

Combining the bounds (10) and (13), we obtain the two-sided limit

$$
-5.8 \times 10^{-12} \leq \tilde{\kappa}_{\mathrm{tr}}-\frac{4}{3} c_{e}^{00} \leq 1.2 \times 10^{-11}
$$

on isotropic deviations of the phase speed of light from the MAS of the electron. This bound represents an improvement of more than 3 orders of magnitude upon previous laboratory-based constraints. We obtained this limit by exploiting the threshold effects of vacuum Cherenkov radiation and photon decay for positive and negative $\tilde{\kappa}_{\text {tr }}$, respectively.

An independent constraint on $\tilde{\kappa}_{\text {tr }}$ may be obtained by future low-energy laboratory tests with estimated sensitivities at the level of $10^{-11}$ or better 28]. Another possibility for improvents may come from photon triple splitting, because the amplitude for this effect is nonzero 
in the presence of $c^{\mu \nu}$-type SME coefficients [29]. As opposed to vacuum Cherenkov radiation and photon decay, photon triple splitting is not a threshold effect, so that it may not necessitate high photon energies.

Significantly improved terrestrial bounds using the same reasoning as presented here may be obtained at the prospective International Linear Collider (ILC). Accelerating electrons to laboratory-frame energies of $500 \mathrm{GeV}$, the ILC may provide a one-sided Cherenkov bound with a sensitivity at the level of $5 \times 10^{-13}$. Similarly, the Large Hadron Collider (LHC) is scheduled to attain roughly seven times the energy of the Tevatron. Assuming that the energy $E_{\gamma}$ of the produced photons scales by the same factor, the bound of Eq. (13) can be sharpened by a factor of 50. Additional improvements of the photon-decay limit may be achieved with a dedicated D0 or LHC study: for instance, the highest-energy data not analyzed for QCD tests could be used for the present purposes. Moreover, the high-energy tail of the photon-energy spectrum could be utilized more efficiently by avoiding large energy binning.

The $\tilde{\kappa}_{\text {tr }}$ limits from both vacuum Cherenkov radiation and photon decay scale quadratically with the energy of the primary particle. At present, UHECR offer the highest possible energies in the Sun-centered celestial equatorial frame; the spectrum is limited only by the opacity of the universe to cosmic rays above certain energy thresholds (e.g., GZK suppression or pair creation on IR-photon background). For example, particles with energies up to about $2 \times 10^{11} \mathrm{GeV}$ have been observed at the Pierre Auger Observatory. Assuming these particles are Iron nuclei, and that the neutron $c_{n}^{\mu \nu}$ coefficients are insignificant, bounds at the $10^{-20}$ level can be extracted [22]. Although this limit is not directly comparable to our results (it does not measure the photon speed relative to the MAS of the electron), it does illustrate the potential of cosmic-ray tests. Primary photons from the Crab nebula are another example: Energies up to $8 \times 10^{4} \mathrm{GeV}$ have been reported by HEGRA [30]. Equation (11) would then give one-sided limits on $\tilde{\kappa}$ coefficients at the level of $10^{-16}$. We mention that at UHECR scales some of the non-birefringent $\tilde{\kappa}$ matrices and certain electron SME coefficients can no longer be neglected, as is apparent by consulting Eq. (5). In any case, the interpretation of future UHECR analyses of Lorentz violation would greatly benefit from a more reliable identification of the primary.

The authors would like to thank B. Altschul for his helpful comments as this work developed, as well as F.R. Klinkhamer and M. Schreck for useful discussions. This work is supported in part by the National Science Foundation and by the European Commission under Grant No. MOIF-CT-2005-008687.
[1] See, e.g., V.A. Kostelecký and S. Samuel, Phys. Rev. D 39, 683 (1989); V.A. Kostelecký and R. Potting, Nucl. Phys. B 359, 545 (1991); S.M. Carroll and J.A. Harvey and V.A. Kostelecký and C.D. Lane and T. Okamoto, Phys. Rev. Lett. 87, 141601 (2001); O. Bertolami and R. Lehnert and R. Potting and A. Ribeiro, Phys. Rev. D 69, 083513 (2004); V.A. Kostelecký, R. Lehnert, and M.J. Perry, Phys. Rev. D 68, 123511 (2003); J. Alfaro, H.A. Morales-Tecotl, and L.F. Urrutia, Phys. Rev. Lett. 84, 2318 (2000); F.R. Klinkhamer and C. Rupp, Phys. Rev. D 70, 045020 (2004); N.E. Mavromatos, Lect. Notes Phys. 669, 245 (2005). J.D. Bjorken, Phys. Rev. D 67, 043508 (2003). N. Arkani-Hamed et al., JHEP 0405, 074 (2004).

[2] D. Colladay and V.A. Kostelecký, Phys. Rev. D 55, 6760 (1997); Phys. Rev. D 58, 116002 (1998); V.A. Kostelecký, Phys. Rev. D 69, 105009 (2004).

[3] R. Bluhm and V.A. Kostelecký, Phys. Rev. D 71, 065008 (2005); R. Bluhm, S.H. Fung, and V.A. Kostelecký, Phys. Rev. D 77, 065020 (2008); V.A. Kostelecký and R. Potting, Gen. Rel. Grav. 37, 1675 (2005).

[4] For recent reviews of various experimental and theoretical approaches to Lorentz violation see, e.g., V.A. Kostelecký, ed., CPT and Lorentz Symmetry I-IV, World Scientific, Singapore, 1999-2008; R. Bluhm, Lect. Notes Phys. 702, 191 (2006) hep-ph/0506054; D.M. Mattingly, Living Rev. Rel. 8, 5 (2005).

[5] V.A. Kostelecký and N. Russell, arXiv:0801.0287.

[6] For a speed-of-light test interpreted outside the SME, see, e.g., V.G. Gurzadyan et al., Mod. Phys. Lett. A 20, 19 (2005).

[7] V.A. Kostelecký and M. Mewes, Phys. Rev. Lett. 97, 140401 (2006).

[8] S. Herrmann et al., in Ref. [4], Vol. IV;

[9] M.A. Hohensee et al., to be published.

[10] This does not necessarily imply causality violations [11]: we can rescale the coordinates such that the new lightcone coincides with the fastest particle [23, 24].

[11] V.A. Kostelecký and R. Lehnert, Phys. Rev. D 63, 065008 (2001).

[12] M.A. Hohensee, R. Lehnert, D.F. Phillips, and R.L. Walsworth, arXiv:0809.3442 [hep-ph].

[13] R. Lehnert, Phys. Rev. D 68, 085003 (2003).

[14] R. Lehnert and R. Potting, Phys. Rev. Lett. 93, 110402 (2004); Phys. Rev. D 70, 125010 (2004).

[15] E.F. Beall, Phys. Rev. D 1, 961 (1970).

[16] S.R. Coleman and S.L. Glashow, Phys. Rev. D 59, 116008 (1999).

[17] F.W. Stecker and S.L. Glashow, Astropart. Phys. 1697 (2001)

[18] T. Jacobson, S. Liberati, and D. Mattingly, Phys. Rev. D 67, 124011 (2003).

[19] B.D. Altschul, Nucl. Phys. B 796, 262 (2008).

[20] B.D. Altschul, Astropart. Phys. 28, 380 (2007).

[21] F.R. Klinkhamer and M. Risse, Phys. Rev. D 77, 117901 (2008).

[22] F.R. Klinkhamer and M. Schreck, Phys. Rev. D 78, 085026 (2008).

[23] V.A. Kostelecký and M. Mewes, Phys. Rev. Lett. 87, 251304 (2001); Phys. Rev. D 66, 056005 (2002).

[24] Q.G. Bailey and V.A. Kostelecký, Phys. Rev. D 70, 
076006 (2004).

[25] LEP Energy Working Group, R. Assmann et al., Eur. Phys. J. C 39, 253 (2005).

[26] D0 Collaboration, V.M. Abazov et al., Phys. Lett. B 639, 151 (2006) [Erratum-ibid. B 658, 285 (2008)].

[27] D0 Collaboration, V.M. Abazov et al., arXiv:0804.1107 [hep-ex].
[28] M.E. Tobar et al., Phys. Rev. D 71, 025004 (2005) [Erratum-ibid. D 75, 049902 (2007)].

[29] V.A. Kostelecky and A.G.M. Pickering, Phys. Rev. Lett. 91, 031801 (2003).

[30] The HEGRA Collaboration, F. Aharonian et al., Astrophys. J. 614, 897 (2004). 